\title{
Optimization of a mercury jet target for a neutrino factory or a muon collider
}

\author{
X. Ding, ${ }^{1, *}$ J. S. Berg, ${ }^{2}$ D. Cline, ${ }^{1}$ and H. G. Kirk ${ }^{2}$ \\ ${ }^{1}$ University of California at Los Angeles, Los Angeles, California 90095, USA \\ ${ }^{2}$ Brookhaven National Laboratory, Upton, New York 11973, USA
}

(Received 14 December 2010; published 9 November 2011)

\begin{abstract}
A study of target parameters for a mercury jet target for a neutrino factory or muon collider is presented. We simulate particle production initiated by incoming protons with kinetic energies between 2 and $100 \mathrm{GeV}$. For each proton beam kinetic energy, we maximize production by varying the geometric parameters of the target: the mercury jet radius, the incoming proton beam angle, and the crossing angle between the mercury jet and the proton beam. With an $8-\mathrm{GeV}$ proton beam, we study the variation of meson production with the entry direction of the proton beam relative to the jet. We also examine the influence on the meson production by the focusing of the proton beam. The number of muons surviving through the neutrino factory front end channel is determined as a function of the proton beam kinetic energy.
\end{abstract}

DOI: 10.1103/PhysRevSTAB.14.111002

PACS numbers: 29.20.db, 29.27.Eg, 29.25.Pj, 13.20.Cz

\section{INTRODUCTION}

Future facilities based on intense beams of muonsthe muon collider and the neutrino factory-offer the promise of extraordinary physics capabilities $[1,2]$. The muon collider (MC) provides a possible approach to a multi-TeV lepton collider, and hence a way to explore new physics beyond the reach of present colliders. In addition, a neutrino factory (NF) gives the opportunity to perform extremely sensitive neutrino oscillation experiments while also opening expanded avenues for the study of new physics in the neutrino sector.

$\mathrm{MC}$ and NF accelerator complexes are shown schematically in Fig. $1[1,3,4]$. Both the MC and NF require a target solution that can convert a multi-MW proton beam into an intense muon source. The requirements for a muon collider and a neutrino factory capable of delivering a large number of muons in order to achieve acceptable performances pose significant challenges to a target. For instance, the target must be a high- $Z$ material in order to produce pions copiously, yet not be so large as to result in a significant rate of absorption of secondary pions within the target material. Second, the target system must survive in the extreme conditions of a powerful MW-class proton beam, where the target will have to dissipate large amounts of energy, survive the strong pressure waves induced by the short beam pulses, and also survive long-term effects of radiation damage. A concept of utilizing a free-flowing mercury

\footnotetext{
*Corresponding author. xding@bnl.gov.
}

Published by the American Physical Society under the terms of the Creative Commons Attribution 3.0 License. Further distribution of this work must maintain attribution to the author(s) and the published article's title, journal citation, and DOI. jet target has been proposed to accomplish the task [5]. The MERIT collaboration [6] built such a target and tested it with an intense proton beam. The experiment validated the concept of a free mercury jet inside a highfield solenoid magnet as a target for a pulsed proton beam of 4-MW power. Figure 2 shows a schematic of the target concept. The target is inside solenoids that generate a 20- $\mathrm{T}$ magnetic field that tapers to lower values of $1.5 \mathrm{~T}$ for $z>15 \mathrm{~m}$ [5]. Pions are produced when a proton beam is delivered onto the mercury jet target. The pions are captured in the high-field solenoids and then transported into a decay channel in which the pions decay to produce both positive and negative muons.

The production of pions at the target depends on the size of the target, the orientation of the proton beam relative to the magnetic field, and the angle of the beam and jet relative to each other. In the present paper, we will report our efforts on the meson production simulations for this mercury jet target to provide an optimized solution for the conversion of a powerful MW-class proton beam into an intense muon beam. The meson production is defined to be the collection of charged muons and charged pions at the end of a $50 \mathrm{~m}$ decay channel. In this paper, Sec. II describes an optimization study of these geometric target parameters for the liquid mercury jet target system and the meson production efficiencies as a function of the primary proton kinetic energy. In Sec. III, we examine the correlation between meson production and multiple beam entry points for the proton beam onto the jet. In Sec. IV, we present the relation between the $\beta^{*}$ of the focused proton beam and the resulting meson production. In Sec. V, we determine the number of muons surviving through the NF front end channel as a function of the proton beam energy and, finally, we conclude with a summary in Sec. VI. 

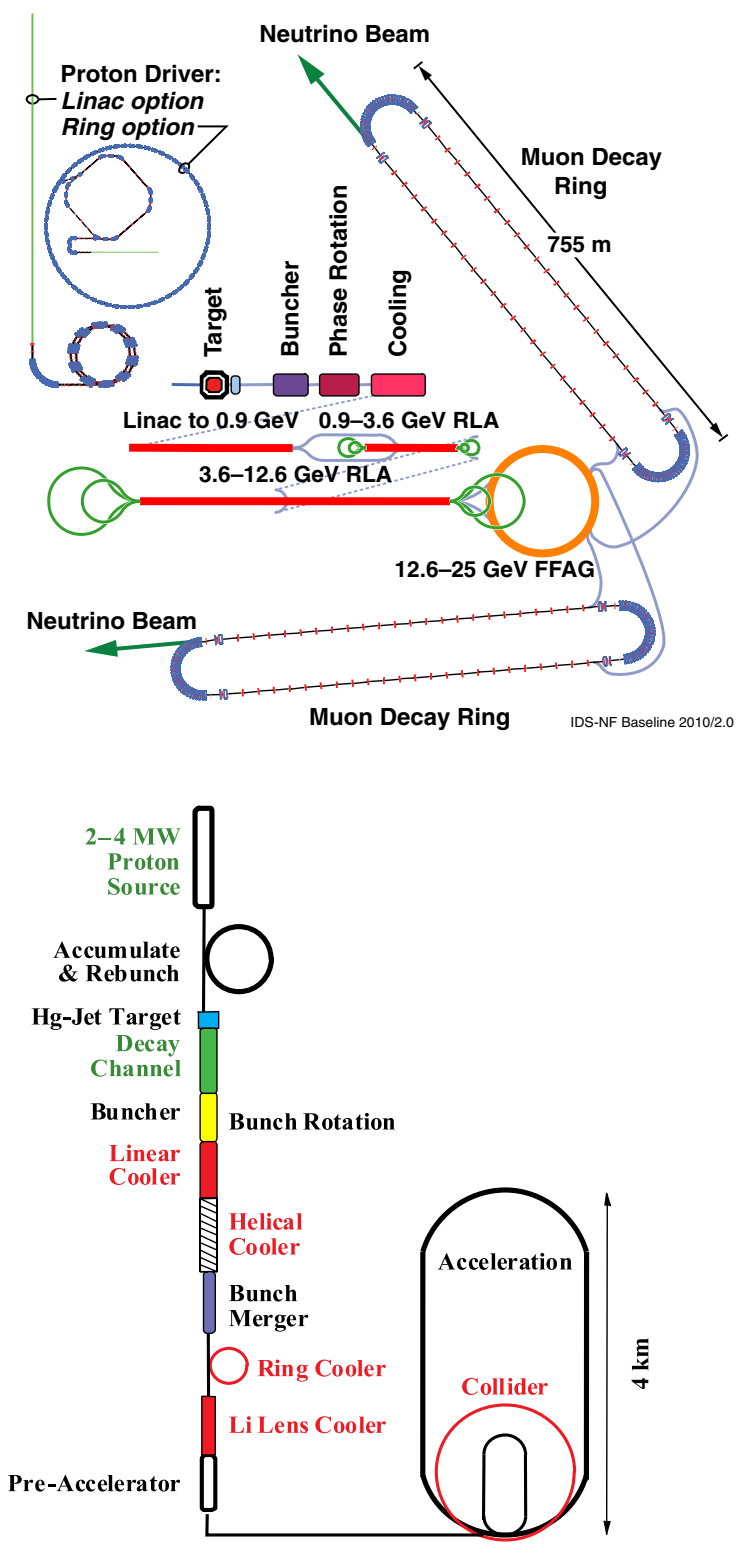

FIG. 1. Schematic of a 25-GeV NF (top); schematic of a 1.5-TeV MC (bottom).

\section{OPTIMIZATION OF GEOMETRIC TARGET PARAMETERS AND MESON PRODUCTION EFFICIENCY AS A FUNCTION OF PROTON BEAM KINETIC ENERGY}

The mercury jet target geometry is shown in Fig. 3. For this series of simulations the proton beam is launched under the mercury jet at $z=-75 \mathrm{~cm}$. The proton beam and mercury jet will intercept at $(0 \mathrm{~cm}, 0 \mathrm{~cm},-37.5 \mathrm{~cm})$. We run MARS15 [7], a Monte Carlo code capable of simulating particle interactions and transport, and count all the mesons that cross a transverse plane 50 meters downstream from the beam/jet interaction. We then select the muons whose kinetic energies are in the range of 40 to $180 \mathrm{MeV}$. This is done because we find that the number of mesons in

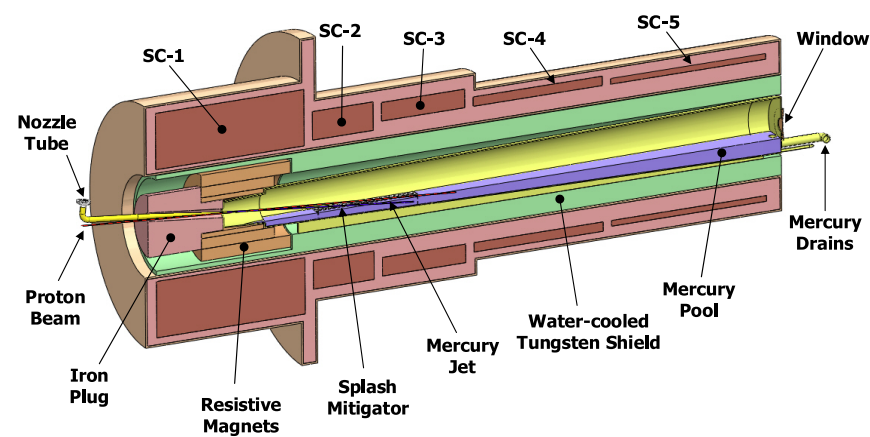

FIG. 2. Concept of a 4-MW target station with a continuous mercury jet target for an intense proton beam. The mercury jet is tilted by $100 \mathrm{mrad}$ with respect to a $20-\mathrm{T}$ solenoid magnet that captures and conducts low-momentum pions into a decay channel. The mercury is collected in a pool that also serves as the proton beam dump.

this kinetic energy band is proportional to the number of mesons which eventually survive the subsequent downstream phase rotation and transverse cooling sections, independent of other parameters such as the proton beam kinetic energy and target geometry [8].

To find the optimal values for the geometric parameters, we first make several simulations with different values for a single parameter. We then fit a curve (using least-squares fitting with a third order polynomial) through the data for meson production (with uncertainties, which can be computed from the MARS15 results since each incoming beam proton is independent) as a function of the parameter in question. We take the parameter value at the maximum in the fitted curve to be the current optimal value for that parameter. We repeat this process for each parameter, and then cycle through the parameters again until the parameter values have converged. As a result of the least-squares fitting process, we are able to obtain uncertainties in the optimal parameter values and the production values.

The parameters we vary are shown in Fig. 4. They are the jet radius, the angle between the proton beam and the magnetic field at $z=-75 \mathrm{~cm}$, and the angle between the jet and the proton beam at their intersection point, $z=$ $-37.5 \mathrm{~cm}$. The proton beam is Gaussian, with an rms radius set to 0.3 times the target radius. When the proton beam angle relative to the magnetic field is adjusted, care is

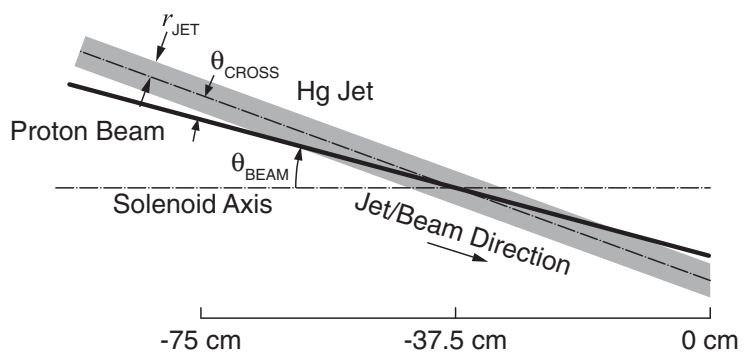

FIG. 3. The mercury jet target geometry. The proton beam and mercury jet cross at $z=-37.5 \mathrm{~cm}$. 
taken to ensure that the launch positions and directions at $z=-75 \mathrm{~cm}$ are properly set so that the beam/jet intercept is always at $x=y=0 \mathrm{~cm}$. For each energy, we start with the proton beam at an angle of $67 \mathrm{mrad}$ with respect to the magnetic field, a crossing angle of 33 mrad between the jet and the proton beam, and a target radius of $5 \mathrm{~mm}[5,9]$.

Using the described optimization method (an example shown in Fig. 5), we get the optimal values and the uncertainties for target radius, beam angle at $z=-75 \mathrm{~cm}$,
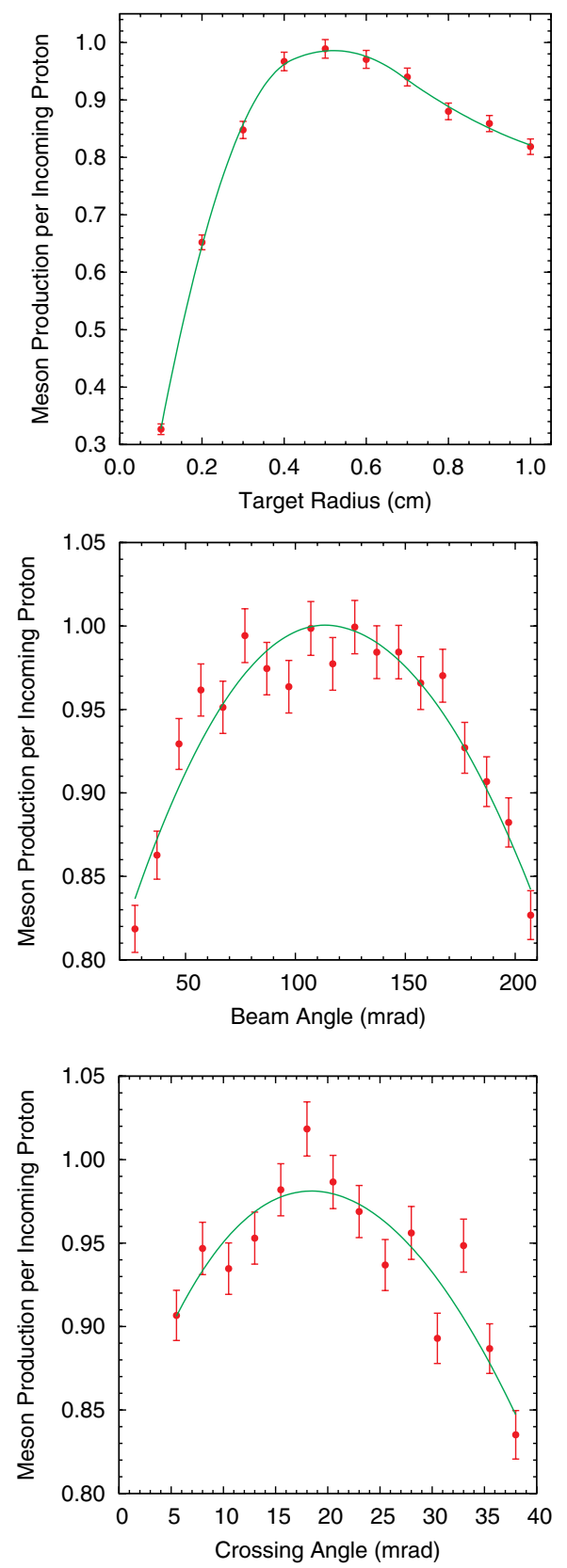

FIG. 4. Meson production as a function of target radius (top), proton beam angle (middle), and beam-target crossing angle (bottom). Data points represent meson production generated from $10^{5}$ incoming $50-\mathrm{GeV}$ protons. Curves are least-squares fits to this data.
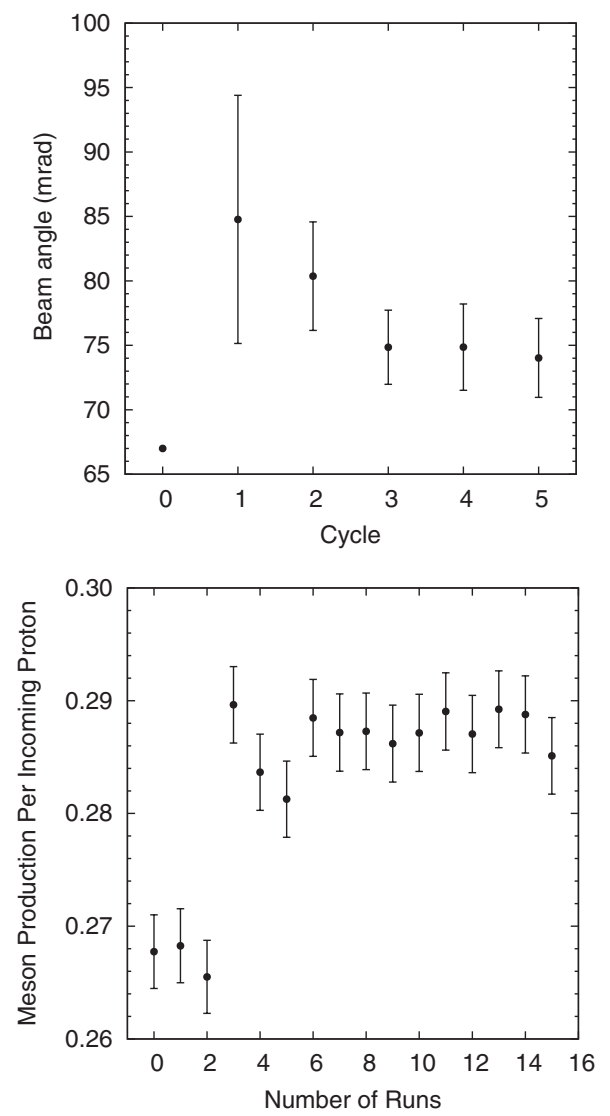

FIG. 5. The optimized proton beam angle in each cycle (top) and meson production as a function of the iteration number during the optimization procedure (bottom). The meson production corresponding to the optimized proton beam angle in each cycle is run at No. 2, No. 5, No. 8, No. 11, and No. 14. Data points are generated from $2 \times 10^{5}$ incoming $8-\mathrm{GeV}$ protons.

and crossing angle at the beam/jet intercept point of $z=-37.5 \mathrm{~cm}$ for proton kinetic energies between 2 and $100 \mathrm{GeV}$. These results are shown in Fig. 6. Figure 7 shows meson production before and after optimization for proton energies between 2 and $100 \mathrm{GeV}$. Each curve has been normalized to the beam power. We see that the meson production is most efficient near proton beam kinetic energies of $8 \mathrm{GeV}$.

\section{MULTIPLE PROTON BEAM ENTRY DIRECTIONS}

In the previous section, the proton beam is launched beneath the mercury jet as shown in Fig. 3. Alternative proton beam entry points are also possible. We consider now the case of an $8 \mathrm{GeV}$ proton beam and compare meson production rates for each entry point. Based on the results depicted in Fig. 6, we fix the mercury jet radius to be $0.4 \mathrm{~cm}$ and the beam/jet crossing angle at $z=-37.5 \mathrm{~cm}$ to be $27 \mathrm{mrad}$. The mercury jet angle to solenoid axis is $96.7 \mathrm{mrad}$. In addition, we define a roll angle to describe on 

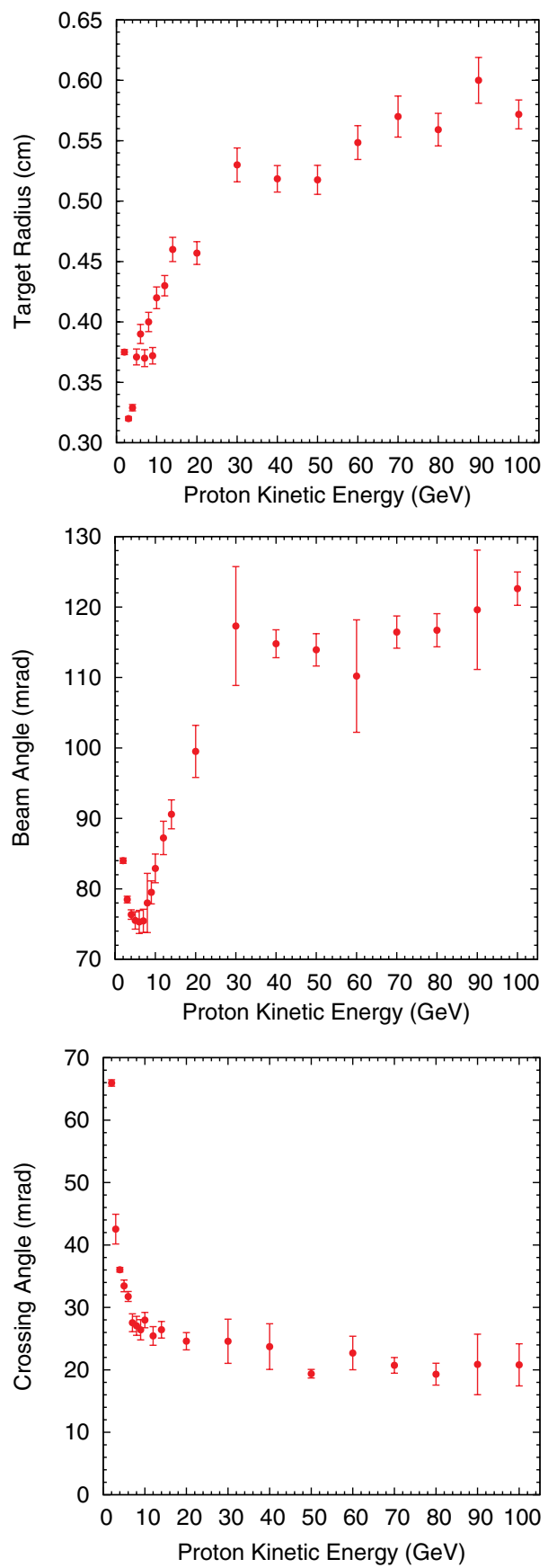

FIG. 6. Optimized target parameters of target radius (top), beam angle at $z=-75 \mathrm{~cm}$ (middle), and beam/jet crossing angle at $z=-37.5 \mathrm{~cm}$ (bottom) as a function of proton kinetic energy.

which side of the jet the proton beam approaches. The roll angle is defined to be 0 if the proton beam enters the target from above.

We define the clearance to be the distance between the proton beam center and the surface of mercury jet target divided by the rms radius of proton beam. Figure 8 depicts the calculated proton beam positions at $z=-75 \mathrm{~cm}$ for 15 cases each of which keep the same crossing angle of

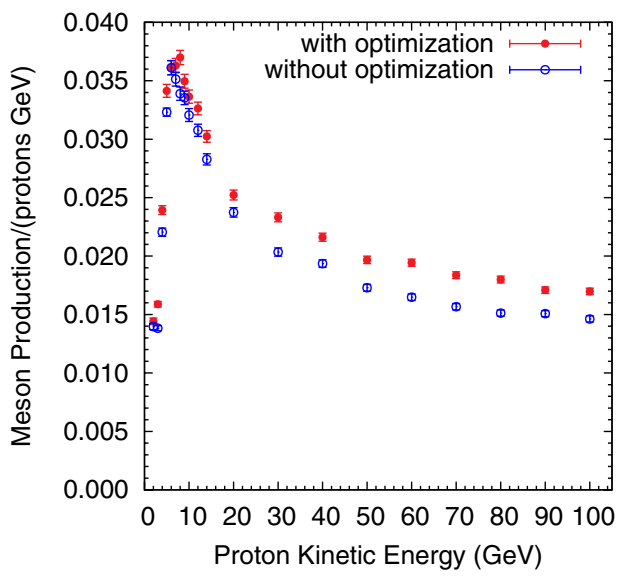

FIG. 7. Production with original geometry and with optimized geometry.

$27 \mathrm{mrad}$ at $z=-37.5 \mathrm{~cm}$. The proton beams are not all the same distance from the jet at $z=-75 \mathrm{~cm}$ since the proton beam is bent by the magnetic field. For example, at $z=-75 \mathrm{~cm}$, the $(x, y)$ coordinates of the mercury jet, $\mathrm{p} 0$ and $\mathrm{p} 7$ are $(0,3.64),(-0.56,4.62)$, and $(-0.103,2.638)$, respectively, in units of $\mathrm{cm}$. All entry directions of the proton beam and mercury jet at $z=-75 \mathrm{~cm}$ are inclined relative to the solenoid axis. The incident beam orientation used in the previous optimization study for the $8 \mathrm{GeV}$ case (grey spot in Fig. 8) is very close to the p6 case. Figure 9 shows the trajectory of the beam relative to the mercury jet in the $x-y$ plane as it moves in $z$, for various initial beam positions at $z=-75 \mathrm{~cm}$. Figure 10 shows the clearance and the meson production for various roll angles. We see a

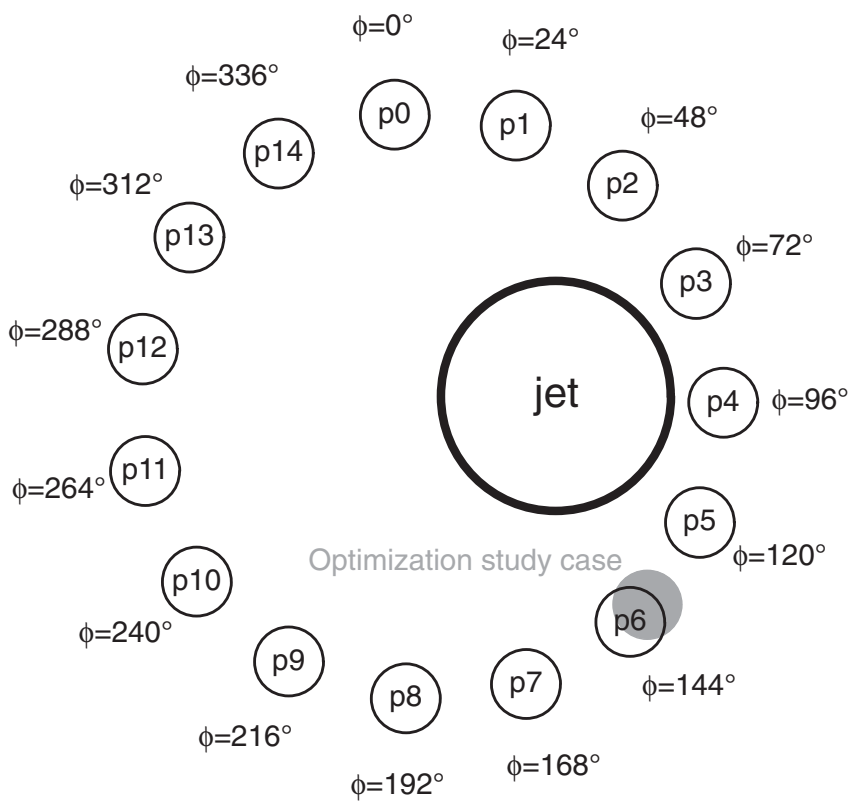

FIG. 8. The layout of multiple proton beam entry directions relative to the mercury jet at $z=-75 \mathrm{~cm}$. 


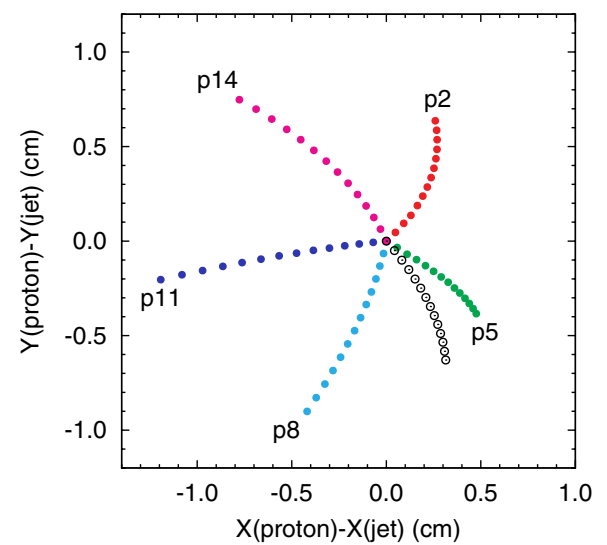

FIG. 9. The trajectory of the $8 \mathrm{GeV}$ proton beam relative to the mercury jet in the $x-y$ plane as the proton moves in $z$. Points on the outside start at $z=-75 \mathrm{~cm}$ at the labeled positions around the beam (see Fig. 8), and head toward a crossing with the jet at $z=$ $-37.5 \mathrm{~cm}$ (convergence of points in the diagram). Open circles are for the direction for which optimization studies were performed.
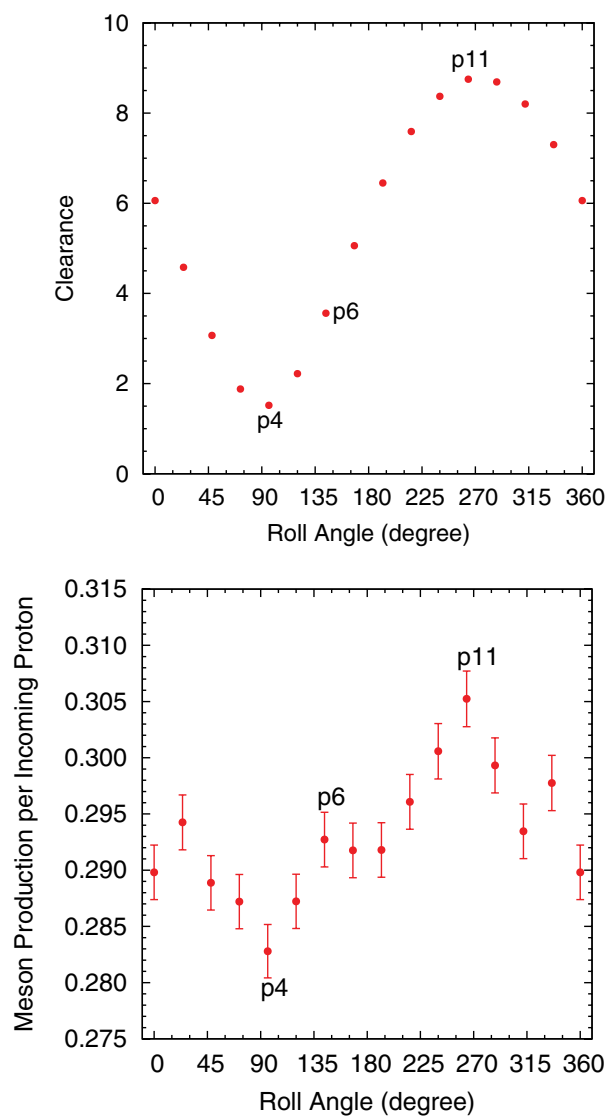

FIG. 10. The clearances (top) and meson production (bottom) for multiple proton beam entry directions relative to the mercury jet at $z=-75 \mathrm{~cm}$. Data points for meson production are generated from $4 \times 10^{5}$ incoming $8-\mathrm{GeV}$ protons.

correlation between the clearance and the meson production. The peak meson production is $8 \%$ higher than for the lowest case and about $4 \%$ higher than for the original case with the proton beam entering from below the jet.

\section{FOCUSED INCIDENT PROTON BEAM}

Our simulations are based on a simple Gaussian incident proton beam with an infinitely large Courant-Snyder $\beta$ parameter. We consider now a focused $8-\mathrm{GeV}$ proton beam and study the correlation between the $\beta$ function of the proton beam and the generated mesons.

In Fig. 11 we show the transverse dimensions of the incident proton beam with a normalized emittance of $25 \pi \mathrm{mm}$-mrad at three longitudinal positions for the case of horizontal and vertical $\beta$ functions of $10 \mathrm{~cm}$ at $z=$ $-37.5 \mathrm{~cm}$. We can clearly see the beam is focused and the beam waist is at $z=-37.5 \mathrm{~cm}$. Figure 12 shows the meson production as a function of $\beta^{*}$ of the proton beam. We see that the meson production loss is negligible $(<1 \%)$ for $\beta^{*} \geq 0.3 \mathrm{~m}$.

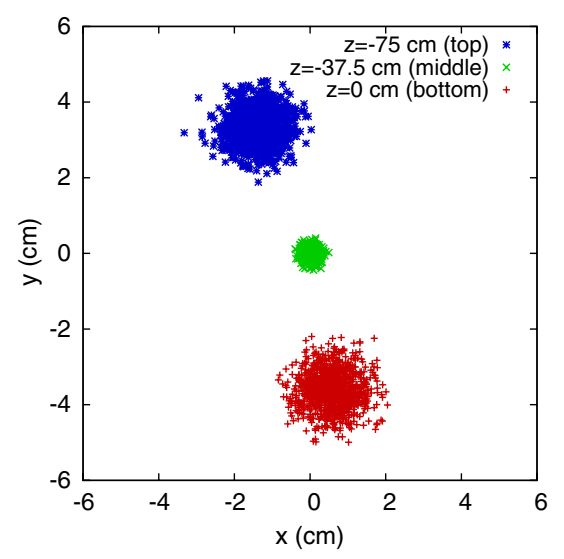

FIG. 11. The $x-y$ plot at three longitudinal positions $(z=$ $-75 \mathrm{~cm}, z=-37.5 \mathrm{~cm}$, and $z=0 \mathrm{~cm}$ ) with an incident proton beam of normalized emittance $25 \pi \mathrm{mm}$-mrad and Twiss parameters of $\alpha_{x}=\alpha_{y}=0, \beta_{x}=10 \mathrm{~cm}$, and $\beta_{y}=10 \mathrm{~cm}$ at $z=-37.5 \mathrm{~cm}$.

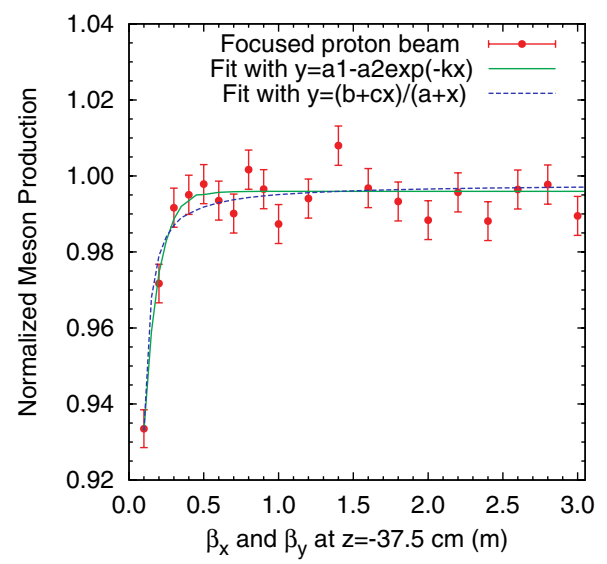

FIG. 12. Meson productions with a Gaussian proton beam, as a function of $\beta_{x}$ and $\beta_{y}$ at $z=-37.5 \mathrm{~cm}$. $\beta_{x}$ is always set equal to $\beta_{y}$ for these simulations. 


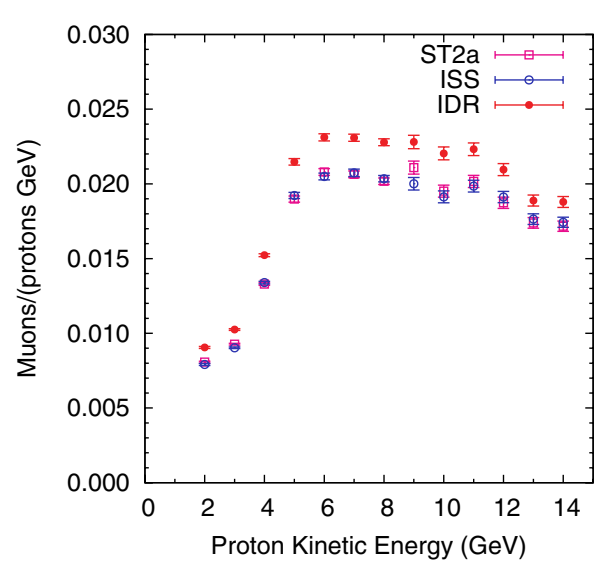

FIG. 13. Muon transmission through the NF front end channel as computed by ICOOL, starting from meson distributions at the downstream end of the target $(Z=0 \mathrm{~cm})$.

\section{MUON SURVIVAL THROUGH THE NF FRONT END CHANNEL}

We compute the number of muons surviving the subsequent phase shaping system for a neutrino factory using version 3.20 of the code ICOOL [10]. We compare the cooling channel performance in the three muon front end lattices (ST2a-BNL for Feasibility Study 2A [11], ST2aISS for the International Scoping Study [12], and IDR for the International Design Report of the International Design Study) [13]. The number of muons surviving as a function of the proton beam energy is shown in Fig. 13. We see that the IDR front end lattice has an improved transmission over the ST2a-BNL lattice and the ST2a-ISS lattice. In addition, for constant beam power, the yield is maximum for a beam energy around $7 \mathrm{GeV}$, but it is within $10 \%$ of this maximum for $5<\mathrm{KE}<12 \mathrm{GeV}$. This result is consistent with the analysis of a tantalum target by Strait et al. [14] in which the cross-section data from the HARP experiment [15] was considered.

\section{CONCLUSIONS}

We optimize the mercury jet target parameters: the mercury jet radius, the incoming proton beam angle, and the crossing angle between the mercury jet and the incoming proton beam with varying kinetic energies. The optimized target radius varies from about 0.35 to $0.6 \mathrm{~cm}$ as the proton beam energy increases. The optimized beam angle at $z=-75 \mathrm{~cm}$ varies from 75 to $120 \mathrm{mrad}$. The optimized crossing angle at $z=-37.5 \mathrm{~cm}$ is near $20 \mathrm{mrad}$ for energies above $10 \mathrm{GeV}$. These values differ from earlier choices of $67 \mathrm{mrad}$ for the beam angle and $33 \mathrm{mrad}$ for the crossing angle. These new choices for the beam parameters increase the meson production by about $20 \%$ compared to the earlier parameters. Our study demonstrates that the maximum meson production efficiency per unit proton beam power occurs when the proton kinetic energy is in the range of $5-15 \mathrm{GeV}$.
An examination of multiple proton beam entry directions relative to the mercury jet for the $8-\mathrm{GeV}$ proton beam case demonstrates that an asymmetric layout is required in order to achieve the same beam/jet crossing angle at the jet axis. The peak meson production is $8 \%$ higher than for the lowest case and about $4 \%$ higher than for the original case with the proton beam entering from below the jet.

An examination of the influence on the meson production by the focusing of the proton beam shows the meson production loss is negligible $(<1 \%)$ for a $\beta^{*}$ of $0.3 \mathrm{~m}$ or higher for the proton beam.

Finally, a simulation of muon transmission through the neutrino factory phase rotation and ionization cooling channel shows that the highest meson production efficiency occurs for proton kinetic energies between 5 and $12 \mathrm{GeV}$.

\section{ACKNOWLEDGMENTS}

This publication is based upon work support by the U.S. Department of Energy in part under Awards No. DEAC02-98CH10886 (BNL) and No. DF-FG02-92ER40695 (UCLA).

[1] Stephen Geer and Michael S. Zisman, Report No. FERMILAB-TM-2459-APC, 2010, https://mctf.fnal .gov/mapproposal.pdf/view.

[2] A. Bandyopadhyay et al., Rep. Prog. Phys. 72, 106201 (2009).

[3] M. Apollonio et al., JINST 4, 07001 (2009).

[4] S. Choubey et al. (IDS-NF collaboration), Report No. IDS-NF-017, 2010) [https://www.ids-nf.org/].

[5] S. Ozaki, R. B. Palmer, M. S. Zisman, and J. C. Gallardo, Report No. BNL-52623, 2001.

[6] K. T. McDonald et al., in Proceedings of the 23rd Particle Accelerator Conference, Vancouver, Canada, 2009 (IEEE, Piscataway, NJ, 2009), p. 795.

[7] N. V. Mokhov, Fermilab-FN-628, 1995; N. V. Mokhov and S. I. Striganov, in Proceedings of the Hadronic Shower Simulation Workshop, Fermilab (2006) [AIP Conf. Proc. 896, 50 (2007)], http://www-ap.fnal.gov/MARS/.

[8] X. Ding et al., in Proceedings of the 23rd Particle Accelerator Conference, Vancouver, Canada, 2009 (Ref. [6]).

[9] J. S. Berg et al., Phys. Rev. ST Accel. Beams 9, 011001 (2006).

[10] R. C. Fernow, in Proceedings of the 18th Particle Accelerator Conference, New York, 1999, edited by C. A. Luccio and W. MacKay (IEEE, Piscataway, NJ, 1999), p. 3020.

[11] M. Alsharo'a et al., Phys. Rev. ST Accel. Beams 6, 081001 (2003).

[12] The International Scoping Study, http://www.hep.ph.ic .ac.uk/iss/.

[13] D. Neuffer et al., in Proceedings of the 2010 International Particle Accelerator Conference, Kyoto, Japan (ICR, Kyoto, 2010), p. 3500.

[14] J. Strait et al., Phys. Rev. ST Accel. Beams 13, 111001 (2010).

[15] M. G. Catanesi et al., Phys. Rev. C 77, 055207 (2008). 